\title{
UMA AMOSTRA DA PRODUÇÃO CIENTÍFICA DA REGIÃO SUDESTE DO BRASIL NO PERÍODO DE 1987-1991
}

\author{
Margarita Antonia Villar Luis* \\ Maria Suely Nogueira* \\ Sebastiana Diniz** \\ Eliene Reis de Oliveira***
}

Realizou-se o presente estudo com o objetivo de investigar acerca da produção científica da região Sudeste no período de 1987 a 1991. para tanto, foram enviados ofícios a 15 escolas de enfermagem das quais tínhamos o endereço ou contatos com pessoas das instituições. Para a inclusão do material no estudo estabeleceram-se alguns critérios considerados importantes para o julgamento e classificação quantitativa dos mesmos. Após este procedimento efetuou-se a classificação qualitativa dos itens a partir dos seus títulos utilizando-se nove categorias temáticas. $O$ volume total de publicações científicas encontradas no período foi de 2237 itens, sendo: 1585 das Escolas de enfermagem do Estado de São Paulo; 222 dos Hospitais da cidade de São Paulo, 296 da Escola de Enfermagem da UFMG e 134 das Escolas de Enfermagem do Rio de Janeiro.

UNITERMOS: pesquisa, enfermagem.

\section{INTRODUÇÃO}

A tradição de desenvolver pesquisa científica na enfermagem brasileira é um fenômeno recente, conforme constatado em CIANCIARULLO \& SALZANO, onde as autoras afirmam que essa atividade teve início no começo do século, há cerca de oitenta anos atrás.

\footnotetext{
* Docente da Escola de Enfermagem de Ribeirão Preto da Universidade de São Paulo.

** Ex-aluna da Escola de Enfermagem de Ribeirão Preto da Universidade de São Paulo.

*** Aluna do $2^{\circ}$ ano de graduação da Escola de Enfermagem de Ribeirão Preto da Universidade de São Paulo.
} 
Desde então os enfermeiros têm elaborado investigações tanto no âmbito da temática de enfermagem em geral como em suas respectivas especialidades de atuação fato que pôde ser constatado a partir do primeiro trabalho documentado, sobre a investigação em enfermagem, "Censo de 1950", mencionado na investigação realizada por NOGUEIRA e cujo conteúdo refere-se a quantidade e tipologia das pesquisas em enfermagem realizadas no Brasil, no período de 1950 a 1981.

A autora utilizou como fontes, os periódicos da época, anais de congressos de

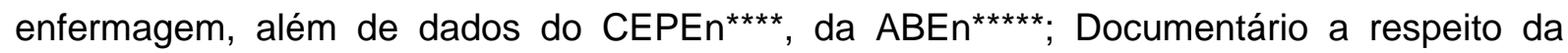
ABEn e Banco de Teses.

TREVIZAN et al. consideram o ano de 1972 como um marco para a pesquisa em enfermagem, uma vez que a partir do mesmo surgiram os cursos de mestrado, desencadeando assim um aumento na produção científica em decorrência da obrigatoriedade de se apresentar, ao fim do curso, uma dissertação.

Com isso, nas últimas décadas tudo leva a supor que o volume de investigações tenha crescido na enfermagem, não só pelo acesso aos cursos de pós-graduação nível mestrado como também devido a implantação do nível doutorado na década de oitenta.

Verificou-se também a participação crescente de enfermeiros assistenciais (particularmente de instituições hospitalares), divulgando seus trabalhos em eventos científicos e em publicações da área da saúde neste período.

Por outro lado, a região Sudeste se constitui numa área geográfica, onde se concentra o maior número de Escolas e Departamentos de Enfermagem, lotados (ou não) em instituições de ensino superior. Também possui o maior número de cursos de pósgraduação, particularmente, de nível Mestrado, contando ainda, com o Doutorado em Enfermagem. Isso tem facilitado que a população autóctone de enfermeiros, tenha o acesso a esses cursos, em vista da proximidade com os locais onde exercem sua atividade profissional.

Devido a essas considerações, as autoras deste estudo, julgam que estimar os itens relacionados à produção científica na região sudeste se constitui, no momento, uma tarefa extremamente laboriosa. É bem verdade que, a produção científica oriunda dos cursos de pós-graduação em enfermagem, é acessível na medida em que há documentos registrando esse material. Da mesma forma, as publicações dos docentes das Escolas de Enfermagem ligadas à Universidade de São Paulo, também estão disponíveis nos relatórios anuais do $\left.\mathrm{SIB|}\right|^{\star \star \star \star \star \star *}$.

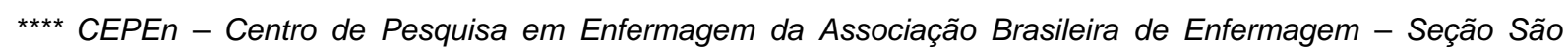
Paulo.

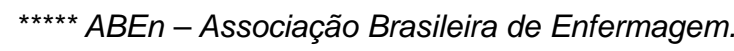

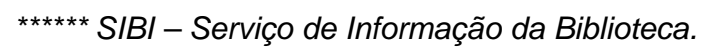


O mesmo não acontece em outras instituições de ensino de Enfermagem, assim como nos serviços assistenciais, obrigando aqueles que queiram obter esses dados a levantá-los em periódicos e anais de eventos, que na atualidade, não se restringem apenas aos exclusivos de enfermagem ou então, entrar em contato com os próprios enfermeiros.

Esse último procedimento foi tentado por FREITAS et al. coordenadora do CEPEn São Paulo, quando as pesquisadoras se propuseram a levantar a pesquisa em São Paulo no triênio 1985-1988. Para tanto, as autoras utilizaram-se do cadastro dos associados da ABEn na seção, tendo obtido o endereço de 1420 profissionais, aos quais foi enviado por correio um formulário contendo itens relativos a produção científica. Desse total de enfermeiros, 1115 (78,5\%) não deram retorno.

Além do baixo índice de resposta $(16,9 \%)$ constatado por FREITAS et al., pode-se acrescentar o fato de que nem todo o enfermeiro é associado à $A B E n$ pois, segundo dados

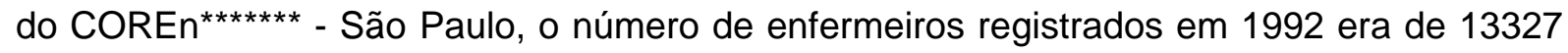
profissionais, enquanto desses, apenas 1420 estavam associados à ABEn.

Como dificuldades adicionais, há de se considerar algumas questões: como a definição do que seja produção científica, os critérios utilizados para selecionar esse material e o que incluir em produção técnico-científica seriam incluídos apenas os itens privilegiados pelos órgãos $\mathrm{CNPq}^{\star \star \star \star \star \star \star \star}, \mathrm{CAPES}^{\star \star \star \star \star \star \star \star \star}$ que existem critérios para a definição do que é produção científica?

A resposta de tais questões parece ser relevante, na medida em que os interessados em obter informações sobre a pesquisa em enfermagem podem ter critérios diferentes na hora de requisitar ou selecionar os dados e por outra, os informantes podem ainda ter uma outra concepção e ainda, não aceitar somente aquela do requisitante.

Cientes dessas dificuldades, quer seja de obtenção de dados, quer seja do entendimento entre os envolvidos, ao aceitar a incumbência de investigar acerca da produção científica da região sudeste, o fizemos no intuito de contribuir para o desvendar desse conhecimento, não tendo a pretensão vã de esgotá-lo.

Para realizar esse empreendimento em tempo tão exíguo, havia dois caminhos: ou centralizavam-se esforços na obtenção de dados provenientes de investigações efetuadas em cursos de pós-graduação, dissertações de mestrado e teses de doutorado e livredocência, ou partia em busca de outras modalidades de pesquisa.

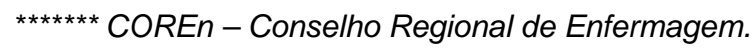

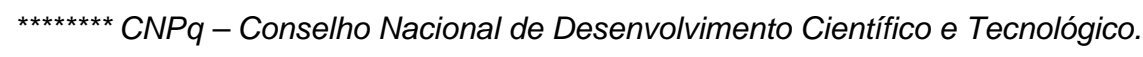

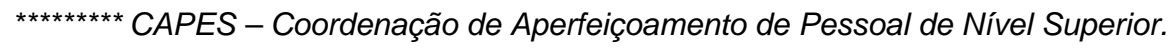


Optou-se pelo segundo caminho, uma vez que satisfazia a curiosidade pessoal das autoras, além de propiciar a oportunidade de serem obtidas informações referentes a produção científica originária de outras fontes. Seguindo esse mesmo raciocínio, julgou-se importante, incluir também na amostra, a produção científica dos enfermeiros dos hospitais.

Assim, o presente estudo tem por objetivo o levantamento da produção científica de uma amostra de instituições de ensino e hospitalares da região sudeste do Brasil no período de 1987 a 1991.

\section{MATERIAL E MÉTODO}

\subsection{Seleção da amostra}

Foi enviado um oficio a uma amostra de 15 escolas de Enfermagem da região sudeste, disponíveis em termos de endereço ou contato pessoal com elementos das instituições. Dessa amostra 10 situavam-se no Estado de São Paulo, sendo 06 no interior e 04 na Capital, respectivamente: Departamento de Enfermagem da Universidade Estadual de Campinas (UNICAMP); Faculdade de Filosofia e Enfermagem Sagrado Coração de Jesus, Faculdade Enfermagem e Obstetrícia de Fernandópolis; Departamento de Enfermagem da Universidade Federal de São Carlos (UFSCAR); Escola de Enfermagem de Ribeirão Preto da Universidade de São Paulo (EERP-USP), Departamento de Enfermagem da Faculdade de Ciências da Saúde Barão de Mauá; Faculdade Enfermagem do Hospital Israelita Albert Einstein; Departamento de Enfermagem da Escola Paulista de Medicina; Faculdade Adventista de Enfermagem e Escola de Enfermagem da Universidade de São Paulo (EEUSP).

No Rio de Janeiro foram contatadas 3 Escolas de Enfermagem, quais sejam: Escola de Enfermagem Anna Nery da Universidade Federal do Rio de Janeiro (UFRJ); Departamento de Enfermagem da Universidade Gama Filho; Faculdade de Enfermagem da Universidade Estadual do Rio de Janeiro (UERJ). Em Minas Gerais enviou-se oficio a Escola de Enfermagem da Universidade Federal de Minas Gerais (UFMG). No Espírito Santo, o contato foi feito via fax com uma docente da Universidade Federal do Espírito Santo, ex-aluna EERP-USP solicitando providências relativas à produção científica de enfermagem do referido estado. Contudo, não houve o envio do material requisitado.

Solicitou-se ainda, auxilio a biblioteca da Escola de Enfermagem da Universidade de São Paulo para o envio de material relativo a mesma e também ao Hospital Universitário da Universidade de São Paulo.

Para a inclusão, do material proveniente de instituições hospitalares, 
solicitou-se a colaboração da presidente da comissão organizadora do IV ENFETEC, bem como a Diretora da Divisão de Enfermagem do Hospital das Clinicas da Faculdade de Medicina de Ribeirão Preto - USP.

O referido ofício foi enviado aos participantes, na semana de 12 a 15 de maio de 1993, solicitando-se que o material relativo à produção científica no período de 1987 a 1991, fosse enviado até o dia 15 de julho de 1993.

Obteve-se resposta de três escolas de São Paulo - Capital (EE-USP, Departamento de Enfermagem da Escola Paulista de Medicina, Faculdade de Enfermagem do Hospital Israelita Albert Einstein) e quatro do interior (Departamento de Enfermagem da UNICAMP, Departamento de Enfermagem da UFSCAR, EERP-USP, Faculdade de Enfermagem e Obstetrícia de Fernandópolis).

Nos demais estados, responderam a solicitação: a Escola de Enfermagem a UFMG, a Escola de Enfermagem Anna Nery (UFRJ), o Departamento de Enfermagem da Universidade Gama Filho e a Faculdade de Enfermagem da UERJ.

Em relação aos hospitais, foram recebidos dados referentes ao Hospital das Clinicas de Ribeirão Preto e de cinco instituições da Capital, quais sejam: Hospital Sírio Libanês, Hospital 9 de Julho, Hospital Alemão Oswaldo Cruz, Hospital da Beneficência Portuguesa e Hospital Unicor.

Deve-se registrar que grande parte do material enviado pelas instituições que participaram do estudo, foi recebido entre os dias 26 de julho a 6 de agosto de 1993.

\subsection{Informações requeridas aos participantes}

Como o estudo consistiu num levantamento da produção cientifica de enfermagem não vinculada a cursos de pós-graduação, solicitou-se aos participantes uma listagem contendo alguns itens contemplados em classificações da produção cientifica elaboradas pelo SIBI e pelo CNPq, os quais foram:

- artigos em periódicos;

- livros e capítulos de livros;

- trabalhos apresentados em congressos e publicados em anais, resumos ou programas;

- trabalhos apresentados em congressos, simpósios e similares (sem publicação).

Além desses itens incluíram-se ainda resenhas, artigos publicados em jornal, fascículos e manuais divulgados a clientelas especificas, os quais acompanhavam o material recebido.

\subsection{Seleção do material}

Para a inclusão neste estudo, das informações enviadas elaboraram-se alguns critérios gerais: 1) verificar se os trabalhos continham dados completos (nome dos autores, data e local de apresentação ou publicação) para serem julgados; 2) observar ainda se as informações se enquadravam no período proposto (1987-1991). 
Como critérios específicos para selecionar o material estabeleceu-se que apresentações em eventos (com ou sem resumo) seriam consideradas uma única vez. E no caso de um mesmo trabalho haver sido apresentado em evento sem resumo e posteriormente em eventos com programa ou anais onde ele se encontrava registrado (resumo), optou-se por classificá-lo no segundo evento.

O mesmo procedimento foi seguido com relação a trabalho apresentado em evento (tendo resumo ou não) e que foi posteriormente publicado; deu-se prioridade a publicação na integra (em período ou anais). Sendo que, quando um mesmo artigo havia sido publicado em mais de um período diferente, considerou-se apenas uma publicação.

Em relação aos livros computou-se o mesmo uma única vez; no caso de mais de um autor, quando não estava especificada a parte que cada um havia elaborado. No caso de, em um único livro, haver a participação de autores em capítulos específicos, considerou-se cada um deles isoladamente.

\subsection{Classificação qualitativa do material}

$\mathrm{Na}$ literatura de enfermagem já existem modelos de categorização da produção científica conforme áreas temáticas, um exemplo disso pode ser constatado nos trabalhos de PELÁ et $\mathrm{al}^{5}$. ALMEIDA et al. $^{1}$ e NOGUEIRA ${ }^{4}$. Tem-se que mencionar ainda, a classificação por áreas do conhecimento elaborada pelos organismos financiadores de recursos para a pesquisa. Com base nela CIANCIARULLO \& SALZANO ${ }^{2}$ elaboraram uma categorização extremamente completa.

Vale ressaltar que atualmente, a diversidade de temáticas estudadas pelas pesquisadoras enfermeiras, vem dificultando imensamente a tarefa de categorizar os trabalhos segundo os temas em estudo.

Para explicar essa variedade, ROCHA \& BOEMER ${ }^{6}$, argumentam que nas pesquisas de enfermagem desenvolvidas no Brasil, há o predomínio da iniciativa individual, uma vez que a pesquisa conduzida por grupos de pesquisadores, é uma alternativa incipiente no Brasil.

Com isso, essas autoras assinalam que as classificações disponíveis estão se revelando insuficientes para contemplar a recente produção científica de enfermagem, uma vez que as aglutinações feitas sob o referencial existente, mostram variações temáticas não tendendo a convergências.

Esse empecilho pode ser, efetivamente, constatado na elaboração do presente estudo, pois na tentativa de classificação dos trabalhos, segundo área temática, verificou-se que as categorias que habitualmente vinham sendo utilizadas em outros estudos eram insuficientes para incluir a variedade de temas inerentes as investigações.

Em função disso, houve a necessidade da adaptação das categorias dos modelos de classificação existentes, modificando-as e inclusive, criando novas categorias que 
possibilitassem a inclusão de todo o material disponível para análise.

Assim sendo, a classificação dos artigos foi feita a partir dos seus títulos, utilizandose de nove categorias elaboradas e descritas a seguir.

\section{Enfermagem}

Estudos referentes a questionamentos sobre a profissão e o profissional enfermeiro analisados sob o enfoque de vários aspectos e abordagens metodológicas; estudos relacionados às teorias de enfermagem; estudos envolvendo aspectos gerais da administração e da assistência geral de enfermagem; estudos enfocando história ética e legislação de enfermagem.

\section{Enfermagem na comunidade}

Estudos relacionados especificamente ao processo saúde-doença focalizando a administração em serviços comunitários; estudos epidemiológicos; atividades assistenciais e/ou implantação e desenvolvimento de programas visando à saúde de promoção, prevenção e reabilitação de pessoas da comunidade.

\section{Enfermagem no hospital}

Estudos envolvendo atuação de enfermagem no âmbito hospitalar e na assistência de enfermagem a pacientes internados; administração específica de serviços, setores e unidades de enfermagem; desenvolvimento de serviços de enfermagem específicos; programas de infecção hospitalar.

\section{Educação e ensino em enfermagem}

Estudos sobre experiências relacionadas ao ensino e a educação em saúde de forma geral; formação de recursos humanos nos vários níveis (médio, graduação e pósgraduação); considerações sobre disciplinas; reflexões sobre currículo (implantação, alterações, sugestões e avaliação); estratégias de ensino; avaliação do processo ensinoaprendizado; levantamento de necessidades e expectativas do aluno.

\section{Enfermagem na pesquisa}

Estudos sob diferentes abordagens filosóficas e delineamentos metodológicos; técnicas e procedimentos de pesquisa em geral; levantamentos e/ou questionamentos sobre a investigação em enfermagem.

\section{Informática em enfermagem}

Estudos sobre a utilização da informática focalizando sua inserção e/ou aplicação na enfermagem; questionamentos relacionados a essa temática. 


\section{Enfermagem do trabalho}

Estudos sobre a enfermagem e o trabalho; assistência a grupos específicos de trabalhadores; implementação de serviços, programas de saúde do trabalhador, investigações em geral sobre doenças relacionadas ao trabalho.

\section{Procedimentos técnicos e tecnologia em enfermagem}

Estudos sobre a aplicação e aperfeiçoamento de procedimentos técnicos de enfermagem; utilização e aperfeiçoamento de instrumental de uso do enfermeiro; manutenção e controle de qualidade desse instrumental; criação de material e instrumentos de apoio aos procedimentos de enfermagem.

\section{Outros}

Estudos abordando temáticas de área do conhecimento não exclusivas da enfermagem ou não direcionadas especificamente a enfermagem.

\section{APRESENTAÇÃO E DISCUSSÃO DOS RESULTADOS}

\section{Total da produção científica da amostra em três estados da Região Sudeste, no período de 1978-1991}

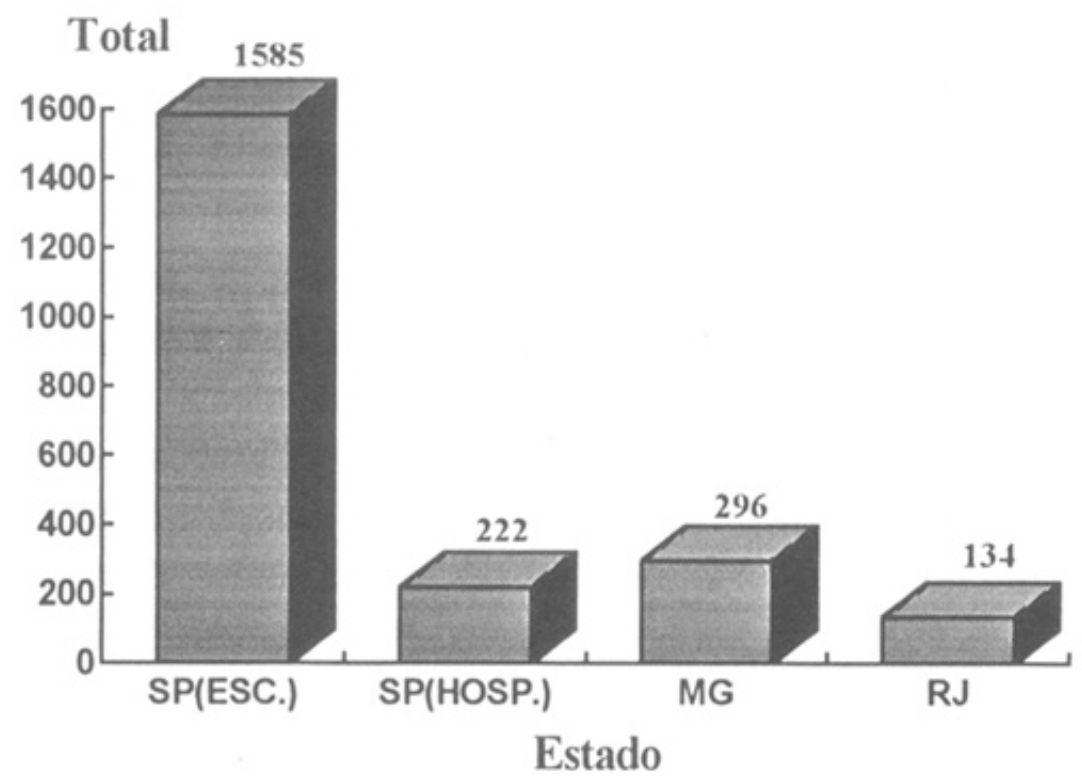

São Paulo: EE-USP e HU/EERP-USP e HCFMRP-USP/Dep. Enf. UNICAMP/Fac. Enf. Obst. Fernandópolis/Dep.

(Escolas) Enf. EPM/Fac. Enf. Hosp. Israelita Albert Einstein/ Dep. Enf. UFSCAR

São Paulo: Hosp. Alemão Oswaldo Cruz/ Hosp. Sirio Libanês/ Hosp. 9 de Julho/ Hosp. Beneficência Portuguesa/ HCFMRP-USP

Rio de Janeiro: Esc. Enf. Anna Nery - UFRJ/ Dep. Enf. Da Univ. Gama Filho/ Fac. Enf. Da UERJ (Escolas) Minas Gerais: Esc. Enf. da UFMG 
No gráfico 1, pode ser observado o volume total de publicações científicas no período - 1987 a 1991, tendo sido encontrados 2237 itens. Verifica-se ainda em termos quantitativos, o predomínio de publicações provenientes das Escolas de Enfermagem do Estado de São Paulo com 1585 itens. É importante registrar que na produção cientifica da EE-USP, estão incluídas 46 publicações elaboradas com a participação de enfermeiros do HU-USP. O mesmo pode ser referido na produção cientifica da EERP-USP onde foram incluídas 53 publicações realizadas em parceria com enfermeiros do HCFMRP-USP.

- Em relação aos hospitais, da cidade de São Paulo e do HCFMRP-USP, constata-se a existência de 222 publicações sem participação de Escolas de Enfermagem.

- Quanto as publicações dos demais estados, foram classificados 296 trabalhos originários da Escola de Enfermagem da UFMG e das escolas de Enfermagem do Rio de Janeiro participantes do estudo (Escola de Enfermagem Anna Nery, Departamento de Enfermagem da Universidade gama filho e Departamentos de Enfermagem da UERJ) evidenciando-se 134 publicações.

\section{Produção científica de uma amostra de instituições da Região Sudeste por ano e por estado - período de 1987 a 1991}

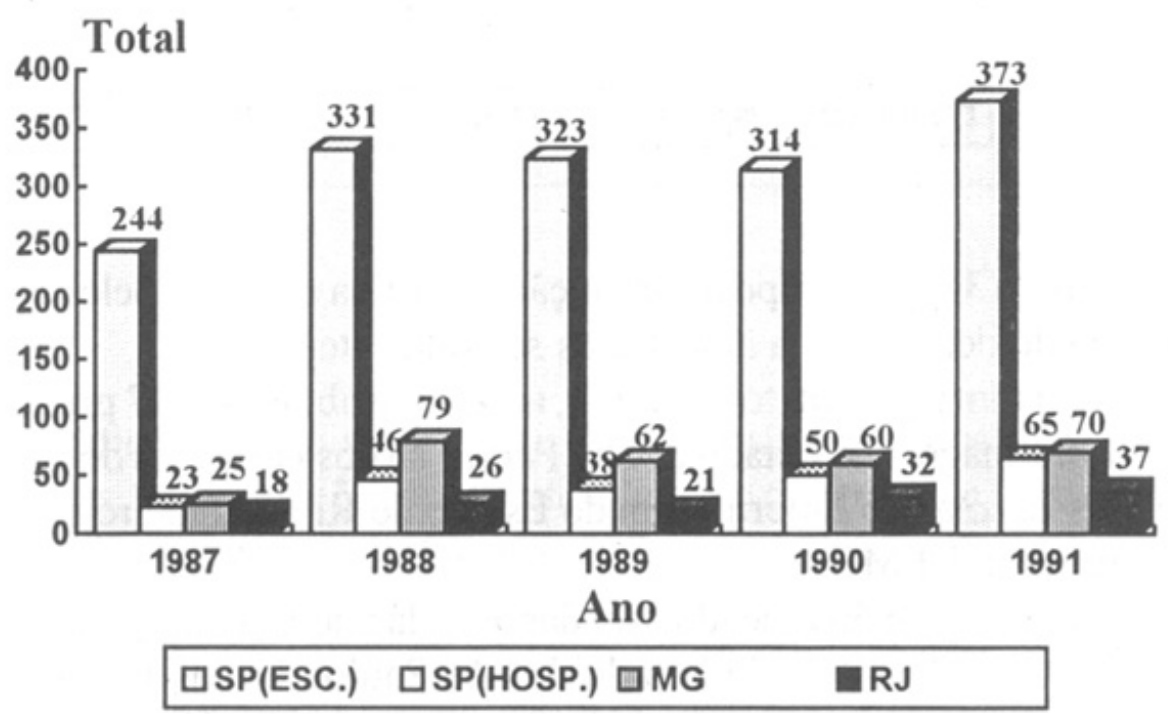

No gráfico 2, podem ser verificados os dados parciais por anos no período, da produção científica, os quais revelam as mesmas informações obtidas a partir do total. Como dado adicional, constata-se que a produção científica na amostra de escolas de São Paulo vem aumentando tanto nas instituições de ensino como nas hospitalares. Verificandose apenas um ligeiro decréscimo em 1990 nas escolas e em 1989 nas escolas e nos hospitais. 
- Em relação à Escola de Enfermagem da UFMG observa-se que apresentou um aumento considerável no ano de 1988, mantendo-se estacionada até 1990, com ligeiro incremento nas suas publicações em 1991.

- Quanto às escolas do Rio de Janeiro que participaram do estudo, os dados indicam uma pequena elevação no ano de 1988 com queda em 1989 e 1990, ressurgindo em 1991.

\section{Tipo de produção científica segundo cada um dos estados da Região Sudeste amostrados - período 1987-1991}

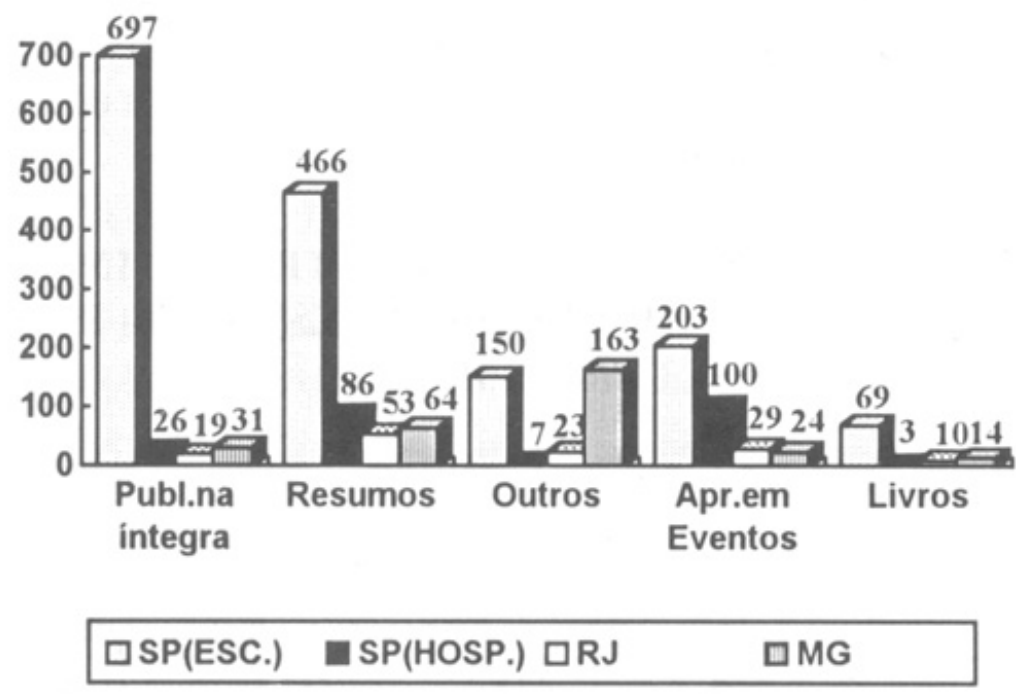

O gráfico 3 indica o tipo de produção científica efetuada pelos participantes do estudo, sendo detectado na amostra os seguintes itens:

- Publicações na íntegra: um total de 773, assim distribuídas: 697 provenientes das Escolas de Enfermagem do Estado de São Paulo; 26 dos Hospitais do Estado de São Paulo; 19 das Escolas de Enfermagem do Estado do Rio de Janeiro e 31 da Escola de Enfermagem da UFMG.

- Resumos: um total de 669, sendo 466 das Escolas de Enfermagem do Estado de São Paulo; 86 dos Hospitais do Estado de São Paulo; 53 das Escolas do Rio de Janeiro investigadas e 64 da Escola de Enfermagem da UFMG.

- Apresentação em eventos: um total de 495, sendo 203 provenientes das Escolas de Enfermagem do Estado de São Paulo; 100 dos hospitais do Estado de São Paulo; 29 das Escolas participantes do Estado do Rio de Janeiro e 163 da Escola de Enfermagem da UFMG.

- Livros e capítulos de livros: um total de 96, sendo 69 originários das Escolas do Estado de São Paulo; 3 dos Hospitais de São Paulo; 10 das Escolas do Estado do Rio de Janeiro e 14 da Escola de Enfermagem da UFMG. 
- Outros: um total de 204, sendo 150 das Escolas do Estado de São Paulo; 7 dos Hospitais de São Paulo; 23 das Escolas de Enfermagem do Estado do Rio de Janeiro e 24 da Escola de Enfermagem da UFMG.

Verifica-se a partir dos dados levantados da amostra estudada, que o Estado de São Paulo, tem predominado, nesse período, em todos os itens referentes a publicação.

\section{Produção científica total de um amostra de instituições da Região Sudeste por área temática (1987-1991)}
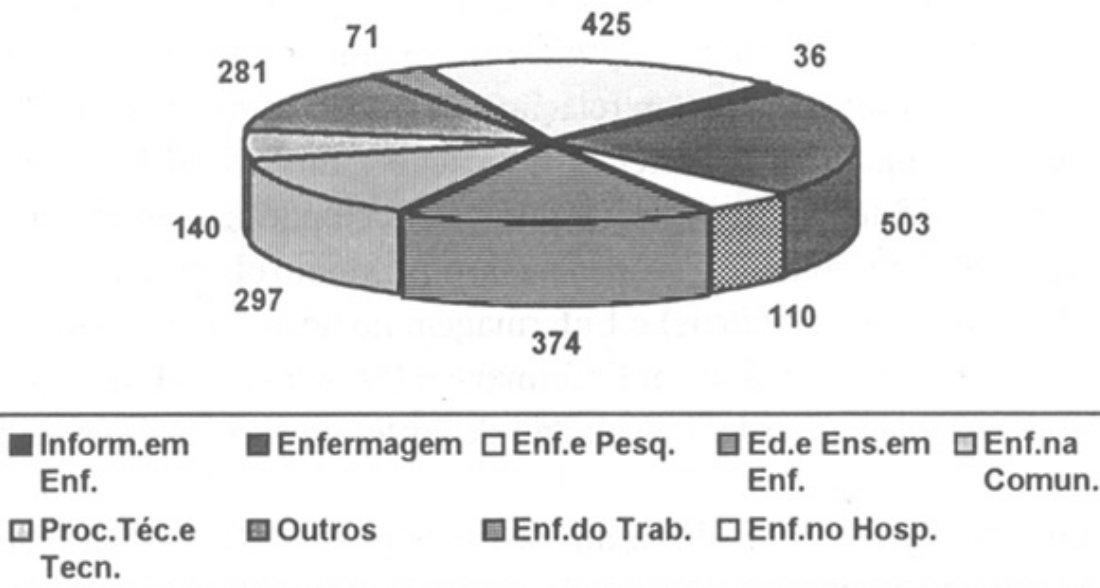

No gráfico 4, pode ser visualizada a classificação dos itens (artigos na íntegra, resumos, apresentações em eventos, livros e capítulos de livros e outros) de produção científica, segundo categoria temática, evidenciando que na amostra estudada, não houve excessiva polarização numa categoria temática, ao menos no que concerne as cinco principais: Enfermagem, Enfermagem no Hospital, Educação e Ensino em Enfermagem, Enfermagem na Comunidade e Outros.

- Verifica-se, que a temática enfermagem predominou em 503 (22,5\%) itens referentes a produção cientifica; seguido de Enfermagem no Hospital, com 374 (16,7\%) itens; Enfermagem na Comunidade, com 297 (13,3\%) itens e finalmente, a categoria "outros" onde foram incluídos $281(12,6 \%)$ itens.

- A categoria Procedimentos Técnicos e Tecnologia em Enfermagem também apareceu em número considerável 140 (6,2\%) itens; seguindo-se de Enfermagem na Pesquisa com 110 (4,9\%). Apareceram ainda, com freqüência bastante reduzida, as 
categorias Enfermagem do Trabalho e Informática em Enfermagem com 71 (3,2\%) e 36 $(1,6 \%)$ itens, respectivamente.

Como informação adicional, foi constatado em relação as principais categorias temáticas que em 1987, predominaram Enfermagem (96 itens), Enfermagem no Hospital (58 itens) e Educação e Ensino na Enfermagem (58 itens), verificando-se a mesma freqüência nas duas últimas categorias. Já, Enfermagem na Comunidade (27 itens) e Outros (23 itens) aparecem em número reduzido na produção cientifica.

Em 1988, observou-se uma inversão dos dados obtidos no tocante as categorias que predominaram, quando considerado o período de 1987-1991, pois no referido ano, houve o predomínio da categoria Enfermagem no Hospital (103 itens) seguida da Enfermagem na Comunidade (82 itens), Educação e Ensino em Enfermagem (80 itens), Enfermagem (66 itens) e por último a categoria Outros (66 itens).

No ano de 1989, observou-se que duas categorias: Enfermagem (103 itens), Enfermagem no Hospital (82 itens), reproduziram a seqüência observada em relação aos dados totais (gráfico 4). Mas em relação às categorias que se seguem, ou seja, Enfermagem na Comunidade (68 itens), Educação e Ensino em Enfermagem (64 itens) e "Outros" (55 itens), houve decréscimo em comparação ao ano anterior.

O ano de 1990 reproduziu a seqüência de predomínio observada em 1989, nas categorias Enfermagem (100 itens) e Enfermagem no Hospital (83 itens), contudo, as categorias Educação e Ensino em Enfermagem (66 itens) e "Outros" (66 itens) mantiveramse na mesma ordem, seguidas de Enfermagem na Comunidade (61 itens).

Constatou-se em 1991, último ano do período estudado, uma nova seqüência de predomínio nas categorias, aparecendo como as mais freqüentes, Enfermagem (125 itens), Educação e Ensino em Enfermagem (107 itens), Enfermagem no Hospital (99 itens).

\section{CONCLUSÕES}

01. Os dados obtidos foram considerados apenas uma amostra da produção cientifica na região sudeste, dada a ausência de resposta de grande parte de instituições de ensino e hospitalares, bem como a ausência da produção de enfermeiros de serviços comunitários.

02. Constatou-se na amostra estudada, que houve acentuadamente, uma maior participação de instituições de ensino e hospitalares do Estado de São Paulo.

03. No que se refere aos hospitais incluídos no estudo, deve-se ressaltar que uma parte considerável do material enviado não pôde ser aproveitada, devido a não contemplar todas as informações solicitadas. 
04. No tocante aos resultados obtidos, ficou evidenciado que, nas Escolas e Departamentos de Enfermagem do Estado de São Paulo, houve o predomínio em todos os itens, de produção cientifica na seguinte seqüência: publicações na integra em periódicos e anais, resumos publicados, onde os dois itens incluem quase metade da produção, apresentação em eventos, outros e por último os livros.

05. Quanto aos hospitais, verifica-se a maior freqüência de trabalhos, no item apresentação em eventos, seguida dos resumos e em número bem menor encontrou-se as publicações na íntegra. Observa-se ainda, que o índice de produção cientifica tem-se mantido estável no período.

06. No que concerne a produção cientifica da amostra do Estado do Rio de Janeiro, notouse que nas escolas e Departamento de Enfermagem de Instituições de ensino do Rio de Janeiro, houve pequena produção (relativa aos itens investigados) mantendo-se praticamente estável no período, com ligeiro aumento entre os anos de 1990 e 1991, tendo sobressaído o item, resumos publicados.

07. Quanto à Escola de Enfermagem da UFMG, verificou-se, que embora apresente uma produção cientifica pequena, nos itens privilegiados pelo estudo, ela vem crescendo ao longo do período (exceto no ano de 1989), aumente esse, ocorrido em função, particularmente, do item "apresentação em eventos" e a seguir pelos resumos publicados.

08. De forma geral, constatou-se que a menor produção ocorreu no item livros, atestando que os enfermeiros, docentes ou assistenciais, encontram dificuldades para a publicação de seu conhecimento nesse veiculo de divulgação.

09. Quanto às categorias temáticas onde foram classificados todos os itens mencionados, constata-se em relação a outros trabalhos da literatura, que houve um aumento na produção cientifica, referente ao tema "Educação e Ensino em Enfermagem".

10. As categorias "Enfermagem" e "Enfermagem no Hospital" continuam predominando e em relação a esta última, considerou-se que houve um desmembramento nessa temática, culminando no surgimento do tema "Procedimentos Técnicos e Tecnologia em Enfermagem".

11. Quanto à temática "Enfermagem na Pesquisa", "Enfermagem no Trabalho" e "Informática em Enfermagem", estas apareceram na amostra estudada como temas prioritários a serem investigados em vista da sua escassez na classificação dos itens de produção cientifica.

12. Quanto a categoria Enfermagem na Comunidade, constatou-se um incremento considerável na produção cientifica no ano de 1988, verificando-se que a partir daí ela veio decrescendo no período.

13. Quanto aos achados em geral relativos às categorias temáticas, considerou-se oportuno recomendar o incentivo de estudos que privilegiam os temas menos freqüentes. 


\section{A SAMPLE OF SCIENTIFIC PRODUCTION IN BRAZIL'S SOUTHEAST REGION - 1987-1991 PERIOD}

The study's goal was to investigate the scientific production in Brazil's southeast region during 1987 to 1991 period. To do that, letters were sent to 15 nursing schools that we had the address or through personal contact with someone from the institutions, asking a list of their scientific production during the period above stated. In order to be included in the study, the article had to meet some criteria that were established as import for a quantitative evaluation and classification. After that, qualitative evaluation and classification. After that, qualitative classification was done by the article's tile using nine categories. The total of publications found were 2237 articles from which, 1585 were from nursing schools in the state of São Paulo, 222 from hospitals in the city of São Paulo, 296 from Federal University of Minas Gerais' School of Nursing and 134 from nursing schools in Rio de Janeiro.

UNITERMS: research, nursing

\section{UNA MUESTRA DE LA PRODUCCIÓN CIENTÍFICA DE LA REGIÓN SUDESTE DE BRASIL EN EL PERÍODO DE 1987-1991}

El presente estudio se ha realizado con el objetivo de investigar la producción científica de la región sudeste en el período de 1987-1991. Para tanto, fueron enviados oficios a 15 escuelas de enfermería, quienes poseíamos seña para correspondencia o se tenía conocimiento de personas de esas instituciones. Para la inclusión del material en el estudio fueron establecidos criterios considerados necesarios para seleccionar lo y para su clasificación cuantitativa. Después de ese procedimiento se efectuó la clasificación cualitativa de los ítems de investigación computados, a partir de sus títulos utilizando se nueve categorías temáticas. El total de publicaciones científicas encontradas, correspondió a 2237 ítems, siendo: 1585 de Escuelas de Enfermería del Estado de São Paulo; 222 de los hospitales de la ciudad de São Paulo; 296 de la Escuela de Enfermería de la Universidad del Estado de Minas Gerais y 134 de las Escuelas de Enfermería del Estado de Rio de Janeiro.

UNITERMOS: investigación, enfermería. 


\section{REFERÊNCIAS BIBLIOGRÁFICAS}

01. ALMEIDA, M. C. P. et al. A produção do conhecimento na pós-graduação em enfermagem no Brasil. In: CONGRESSO BRASILEIRO DE ENFERMAGEM, 33, Manaus, 1981. Anais. Manaus: Associação Brasileira de Enfermagem, 1981. p. 11926.

02. CiANCIARULlO, T. I., SALZANO, S. D. T. A enfermagem e a pesquisa no Brasil. Rev. Esc. Enfermagem USP, v. 25, n. 2, p. 195-215, ago. 1991.

03. FREITAS, D. M. V., RUFFINO, M. C., SAEKI, T. A produção científica do enfermeiro do Estado de São Paulo no triênio 1985-1988. Rev. Paul. Enfermagem, v. 11, n. 3, p. 123-29, set./dez. 1992.

04. NOGUEIRA, M. J. De C. A pesquisa em enfermagem no Brasil: retrospectiva histórica. Rev. Esc. Enfermagem USP, v. 16, n. 1, p. 17-26, 1982.

05. PELÁ, N.T. R., ALMEIDA, M. C. P., ANGERAMI, E. L. S. Situação atual dos cursos e produção científica da pós-graduação da Escola de Enfermagem de Ribeirão Preto, USP. In: SEMINÁRIO NACIONAL SOBRE ENSINO DE PÓS-GRADUAÇÃO E PESQUISA EM ENFERMAGEM, 2, Brasília, 1982. Avaliação e Perspectiva: Brasília: Conselho Nacional de Desenvolvimento Científico e Tecnológico/Associação Brasileira de Enfermagem, 1982. p. 8-18.

06. ROCHA, S. M. M, BOEMER, M. R. Impacto social da pesquisa em enfermagem. Rev. Esc. Enfermagem USP, v. 26, p. 49-60, 1992. (número especial).

07. TReVIZAN, M. A., MENDES, I. A. C., ANGERAMI, E. L. S. A investigação em enfermagem no Brasil. Rev. Paul. Enfermagem, v. 10, n. 3, p. 91-5, set./dez. 1991. 